\title{
MEDIOS ALTERNATIVOS. Panorama de la normatividad de la televisión comunitaria
}

\author{
Lizandro Angulo Rincón* \\ Heverd Páez Quintana** \\ DOI: https://doi.org/10.33571/revistaluciernaga.v11n22a7
}

\section{Resumen}

En este artículo se reflexiona en torno al panorama de la normativa de Colombia en relación a la televisión comunitaria. Se realiza el análisis de la Resolución 650 de 2018, por medio de la cual se reglamenta este tipo de servicio. Para tal fin, se toma como ejemplo el caso de la Asociación de Usuarios Comunitarios de la Antena Parabólica San Jorge de Ocaña (Asucap San Jorge). Se concluye que hay un contexto normativo adverso para este tipo de medios alternativos.

Palabras clave: Televisión; comunidad; medios; comunicación altenativa.

Recibido. Junio 03, $2019 \quad$ Aceptado. Junio 22, 2019

*Doctor en Comunicación y Máster en Participación y Desarrollo Comunitario, ambos posgrados por la Universidad del País Vasco (España); profesor asociado de la Universidad del Tolima (Colombia) y director del grupo de investigación en Comunicación sobre Ciencia, Tecnología y Sociedad (www.sociedadredut.com). Orcid. https://orcid.org/0000-0001-8954-4116;e-mail: langulo@ut.edu.co ** Magíster en Tecnología Educativa y Medios Innovadores del Tecnológico de Monterrey (México), profesor tiempo completo de la Universidad Francisco de Paula Santander Seccional Ocaña (Colombia), director del grupo de investigación Communis del Plan de Estudios de Comunicación Social. Orcid. https://orcid.org/0000-0002-2153-6360; e-mail: coomunis@ufpso.edu.co 


\title{
ALTERNATIVE MEANS. Overview of Community Television Regulations
}

\author{
Lizandro Angulo Rincón* \\ Heverd Páez Quintana** \\ DOI: https://doi.org/10.33571/revistaluciernaga.v11n22a7
}

\section{Abstract}

This paper reflects on the regulation in Colombia for community television. It analyses Resolution 650 of 2018, which regulates this type of service. To this end, the case of the Association of Community Users of the San Jorge Parabolic Antenna in Ocaña (Asucap San Jorge) is taken as an example. It is concluded that there is an adverse regulatory context for this type of alternative media.

Key words: Television; community; legislation; functioning; sustainability.

Received. June 03, $2019 \quad$ Accepted. June 22, 2019

* $\mathrm{PhD}$ in Communication and Master in Participation and Community Development, both postgraduate degrees from the University of the Basque Country (Spain); associate professor at Universidad del Tolima (Colombia), and director of the research group in Communication on Science, Technology and Society (www.sociedadredut.com). Orcid. https://orcid.org/0000-0001-8954-4116 ;e-mail: langulo@ut.edu.co

** Magister in in Educational Technology and Innovative Media from the Tecnológico de Monterrey (Mexico); full-time professor at the Universidad Francisco de Paula Santander - Ocaña Branch (Colombia); director of the Communis research group of the social communication program. Orcid. https://orcid.org/0000-0002-2153-6360; e-mail: coomunis@ufpso.edu.co 


\title{
MEIOS ALTERNATIVOS.
}

Panorama da regulamentação comunitária em matéria de televisão

\author{
Lizandro Angulo Rincón* \\ Heverd Páez Quintana** \\ DOI: https://doi.org/10.33571/revistaluciernaga.v11n22a7
}

\section{Resumo}

Este artigo reflecte sobre o panorama dos regulamentos na Colômbia em relação à televisão comunitária. Analisa a Resolução 650 de 2018, que regulamenta este tipo de serviço. Com esse fim, toma-se como exemplo o caso da Associação de Utilizadores Comunitários da Antena Parabólica de San Jorge em Ocaña (Asucap San Jorge). Concluise que existe um ambiente regulamentar adverso para este tipo de meios de comunicação alternativos.

Palavras chave: Televisão; comunidade; legislação; funcionamento; sustentabilidade.

Recebido. Junho 03, $2019 \quad$ Aceito. Junho 22, 2019

*PhD em Comunicação e Mestrado em Participação e Desenvolvimento Comunitário, ambos pós-graduados pela Universidade do País Basco (Espanha); professor associado da Universidade de Tolima (Colômbia) e director do grupo de investigação em Comunicação sobre Ciência, Tecnologia e Sociedade (www.sociedadredut.com). Orcid. https://orcid.org/0000-0001-8954-4116 ; e-mail: langulo@ut.edu.co

**Mestrado em Tecnologia Educativa e Meios de Comunicação Inovadores pelo Tecnológico de Monterrey (México); professor titular da Universidade Francisco de Paula Santander Seccional Ocaña (Colômbia); director do grupo de investigação Communis do Plano de Estudos em Comunicação Social. Orcid. https://orcid.org/0000-0002-2153-6360; e-mail: coomunis@ufpso.edu.co 
La televisión comunitaria de Colombia, durante más de 20 años ha contado con un soporte legal, lo cual ha posibilitado su desarrollo en distintas regiones del país, sin embargo con el advenimiento del tratado de Libre Comercio entre Colombia y Estados Unidos y la derogada Resolución 433 de 2013, se le ha restado competitividad, a tal punto que varios de estos sistemas de televisión han desaparecido o han migrado a TV por cable o por internet, lo que supone perdida de su esencia comunitaria sin ánimo de lucro.

Los últimos cinco años han sido difíciles para las televisiones comunitarias $y$, especialmente, para Asucap San Jorge Ocaña, modelo en el país por su estabilidad y excelente programación. El problema radica en que el Estado colombiano la ha puesto a competir en evidente desventaja frente al capital transnacional de la televisión por suscripción y por internet. $\mathrm{Ha}$ beneficiado a las empresas privadas internacionales incluidas en el Tratado de Libre Comercio con Estados Unidos con lo que se ha perjudicado la iniciativa comunitaria audiovisual de la nación que, aparte de generar empleo mediante la contratación de personal y la compra de equipos de recepción, producción y transmisión televisiva, promueve la inclusión y la democracia a través de programación educativa y cultural.

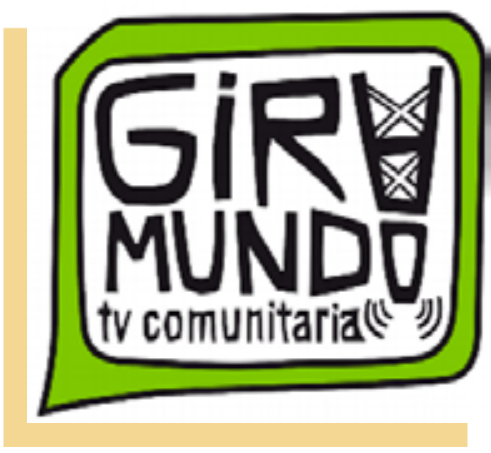

La nueva Resolución, 650 de 2013, es, por ahora, un tímido esfuerzo por reivindicar a las televisiones comunitarias, asfixiadas económicamente por la Resolución derogada, la 433 de 2013, por el incremento de los pagos por compensación y la reducción significativa del número de sus asociados. Asucap San Jorge de Ocaña ha podido subsistir a pesar de esa normativa adversa. Se requiere que se realicen reformas de fondo a la normativa actual que le permita a todas las televisiones comunitarias funcionar y sostenerse en condiciones económicas estables, de modo que puedan mejorar su programación, contratar personal calificado y consolidarse como televisiones que fomente la participación y la acción social para el bienestar comunitario.

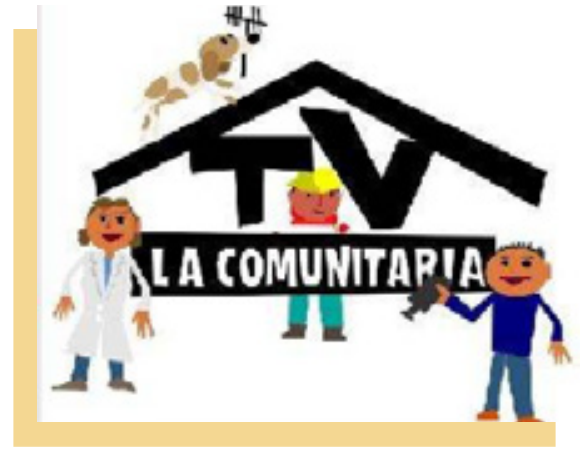

\section{Comunicación para el desarrollo y los medios comunitarios}

En 1950 la propaganda asociada a la influencia del desarrollo extranjero, comienzan a tener forma en los estrados académicos teniendo en cuenta la premisa de que la información tiene un alto poder para el desarrollo. Para MacQuail (2000), a partir de la década del 70 se gestaron medios de comunicación más cercanos a la gente, que atendieran las demandas de los ciudadanos; desde "la teoría se abogó por el derecho a una información local pertinente, a utilizar los media para la interacción y las relaciones sociales en comunidades pequeñas como grupos de interés o subculturas" (p. 211).

Dagron (2012), indica que los medios masivos de información han sido la columna vertebral de las campañas de mercadeo social como paradigma del desarrollo. Valle (2012), considera que los medios alternativos de comunicación, en los que se incluye la 
TV comunitaria, no son sólo emisores de mensajes, sino también generadores de tejido social. La autora indica que:

La comunicación alternativa es la herramienta básica para fortalecer la circulación de información en un grupo determinado, mejorando o superando los efectos que producen los medios de información masivos. Esto quiere decir que la comunicación alternativa no sólo se ocupa de los problemas internos de un grupo, sino que puede trabajar cualquier información de actualidad desde la perspectiva que interesa a los miembros de la comunidad que participan en ella. Puede usarse para ampliar información que no es lo suficientemente desarrollada en los medios masivos, o para propiciar debates y discusiones en la comunidad que permitan a todos comprender mejor lo que suceda en ella misma, en el país o en el mundo (p. 44).

Los medios de comunicación, especialmente los comunitarios, deben estar al servicio de las comunidades, y los grupos de interés deben involucrarse activamente para ejercer presión sobre las decisiones políticas. Todo ello conlleva a que la participación a través de los media, tenga un efecto dominó que involucre la comunidad promotora, los entes territoriales y el público en general (Servaes et al., 1989), (Valle, 2012).

Para Dagron (2002), la TV comunitaria no es el púlpito desde donde los gobiernos se dan propaganda o justifican sus actuaciones políticas, ni quieren convertirse en un pulpo como sucede con las cadenas comerciales, más interesadas en obtener rating a toda costa, con fines económicos. Enfatiza que estos sistemas pretenden tener en cuenta las voces locales y su objetivo no es imponerse a gran escala ni desatar una lucha sin cuartel por las audiencias, sino rescatar el pálpito de la comunidad, el pulso de la vida cotidiana. Angulo (2013) indica que la TV comunitaria se fundamenta en darle participación a la comunidad en los procesos de gestión, producción y control; crean contenidos locales que promueven la inclusión, la fiscalización de la administración pública y el apoyo al cumplimiento de indicadores de desarrollo humano; la transmisión de los valores de la localidad y la convergencia con las tecnologías de la información y la comunicación.

Para García (2012) la televisión regional colombiana surge como una necesidad de expresión cultural de las regiones. La Autoridad Nacional de Televisión de Colombia (ANTV, 2018) [1] define la televisión comunitaria como:

El servicio de televisión cerrada prestado por las comunidades organizadas que tiene como finalidad satisfacer necesidades educativas, recreativas y culturales, y cuya programación tiene un énfasis de contenido social y comunitario. En razón a su restricción territorial y por prestarse sin ánimo de lucro, este servicio no es igual al de televisión por suscripción (p. 8).

La ANTV(2018)también hace referencia a las personas que hacen parte del sistema como asociados y a los canales comunitarios como parte de la difusión, mediante la producción diaria de contenidos que deben "responder a las necesidades de sus asociados... y garantizar el pluralismo informativo de la comunidad organizada a la cual se presta el servicio".

\section{Perfil de la Asociación de Usuarios Comunitarios de la Antena Parabólica San Jorge (Asucap San Jorge)}

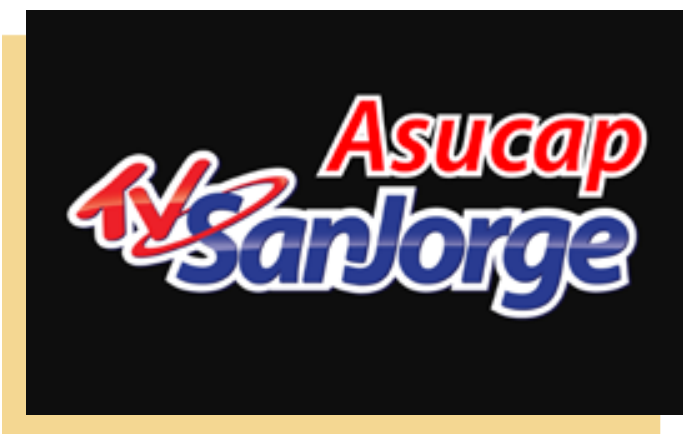

El sistema parabólico San Jorge se fundó en 1989. Dos ingenieros eléctricos Ciro Rodríguez y Raúl Rochel, diseñaron el 
proyecto y luego lo cedieron a la comunidad. En 1991 se creó la Asociación de Usuarios Comunitarios de la Antena Parabólica Asucap San Jorge en Ocaña, Norte de Santander, Colombia, con aproximadamente 700 usuarios.

El Canal Comunitario inicia actividades en 1998. Comenzó la producción propia con un formato informativo local denominado El Noticiero, realizado con periodistas empíricos de la época. Para el año 2000 la Comisión Nacional de Televisión (CNTV) [2], entrega las licencias de funcionamiento y desde entonces comenzó a crecer la parrilla de programación, el personal periodístico y de producción.

Actualmente emiten más de tres horas diarias con tecnología de punta y es considerado un modelo a seguir en el país. Ha obtenido logros importantes como reconocimiento al trabajo de 20 años. Premios como el India Catalina en el 2017 con la mejor producción comunitaria denominada Sequía en el Catatumbo expresan la calidad de la producción.

Tabla 1.

Parrilla de programación de 2018 de Asucap San Jorge Ocaña

\begin{tabular}{|c|c|c|c|}
\hline Programa & Formato & Horario & Productores \\
\hline $\begin{array}{l}\text { Informes } \\
\text { especialos } \\
\text { durante el dia } \\
\text { de } 10 \text { a } 15 \\
\text { minutos en } \\
\text { directo }\end{array}$ & Informativo & $\begin{array}{l}\text { 9-10-11 de la } \\
\text { mah̆ana } \\
12 \text { meridianos. } \\
1 \text { y } 3 \text { de la tarde } \\
\text { de lunes a } \\
\text { viernes }\end{array}$ & $\begin{array}{l}\text { Periodistas } \\
\text { del canal }\end{array}$ \\
\hline $\begin{array}{l}\text { Magazin } \\
\text { Sala } 20\end{array}$ & Contenedor & $\begin{array}{l}\text { De } 4 \text { a } 6 \text { de la } \\
\text { tarde de lunes a } \\
\text { viernes }\end{array}$ & $\begin{array}{l}\text { Periodistas } \\
\text { del canal }\end{array}$ \\
\hline $\begin{array}{l}\text { Noticias Tv } \\
\text { San Jorge }\end{array}$ & Informativo & $\begin{array}{l}\text { De } 6 \text { a 7:30 } \\
\text { p.m. de lunes a } \\
\text { viernes }\end{array}$ & $\begin{array}{l}\text { Periodistas } \\
\text { del canal }\end{array}$ \\
\hline
\end{tabular}

Fuente: Jácome (2019)

Aunque la mayoría de la parrilla es realizada por la producción propia, entidades e instituciones púbicas (Centrales Eléctricas de Norte de Santander y la Universidad Francisco de Paula Santander Ocaña), así como otras comunidades organizadas (colegios), también han producido espacios de corte educativo, principalmente, para dar cumplimiento a la legislación y promover la participación. Además del sistema análogo de televisión, los programas se transmiten a través de Página web y Facebook Live. Vale recalcar que, según el modelo colombiano de TV comunitaria, Asucap San Jorge Ocaña y todos los sistemas de este tipo en el país, ofertan tres tipos de canales: incidentales, codificados y el comunitario, por el que transmiten producción propia local.

Los principales aportes de Asucap San Jorge Ocaña a sus asociados y municipio donde opera son: mantener informada a las personas, ofrecer producción propia de televisión, dar participación a todos los ciudadanos para que expresen sus necesidades, proyectos, expectativas, crear una comunicación directa entre las personas y las autoridades competentes para que se resuelvan los problemas comunitarios, adelantar campañas sociales en temas de vivienda, salud, etc., divulgar la cultura y el patrimonio de la ciudad y ser su garante histórico.

Sus principales dificultades para operar son: mantener al usuario satisfecho con buena programación. Además, pagar la nómina de todos los 50 empleados directos (incluidos los productores de programación propia), el pago de la compensación a la ANTV y, ahora especialmente, el pago de los canales codificados, pues la mayor parte de los recursos se los llevan los derechos de autor de los canales internacionales.

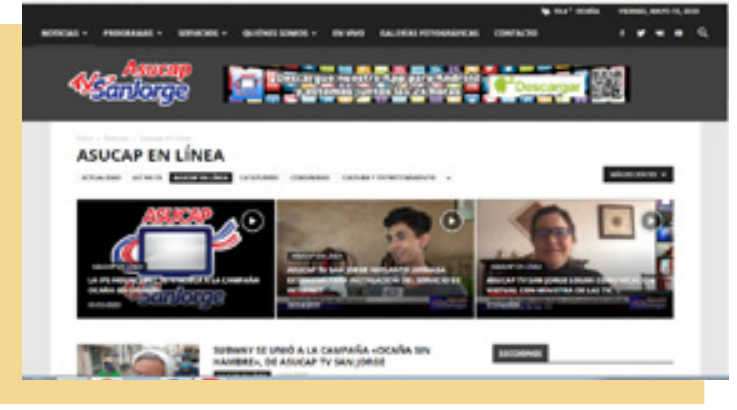

\section{Metodología}

La investigación realizada empleó el estudio de caso, considerado como un enfoque cuantitativo y cualitativo para analizar un fenómeno, en la dinámica del mundo real y en contextos naturales (Weerakkody, 2012). Se hizo revisión documental y se aplicaron entrevista a profundidad. 
La revisión documental supone la selección, acopio, organización y análisis de documentos escritos, audiovisuales, relacionados con un tema específico (Toro y Parra, 2010). La muestra documental analizada fue:

La Resolución 433 de 2013 (derogado), el Acuerdo 009 de 2006 (derogado) y la Resolución 650 de 2018 (vigente), reglamentaciones sobre las televisiones comunitarias de Colombia y el Tratado de Libre Comercio entre Colombia y Estados Unidos (Mincomercio, 2007). Los directorios de TV comunitaria en Colombia de la ANTV (2018), en el que observa el decrecimiento de los sistemas comunitarios en Colombia y el departamento Norte de Santander [3] y el informe del centro de estudios de la Fundación para la Libertad de Prensa (Flip, (2017), que permitió conocer el diagnóstico de la información local en el país.

Se utilizó la entrevista en profundidad, éstas son encuentros reiterados entre el investigador y el entrevistado dirigidos hacia la comprensión de las perspectivas que tienen los informantes respecto a sus vidas, experiencias y situaciones (Soler, 2011). También se realizaron entrevistas semi-estructuradas, las cuales utilizan un cuestionario flexible (Weerakkody, 2009). Los entrevistados debían cumplir con los siguientes criterios: (1) llevar más de un año de trabajo en ASUCAP San Jorge; (2) demostrar conocimiento de la reglamentación derogada y vigente de la televisión comunitaria de Colombia y (3) conocer las dinámicas administrativas y de producción de contenidos/programas de estos sistemas comunitarios.

Los entrevistados fueron seleccionados por conveniencia (Hernández, Fernández \& Baptista, 2010). Las preguntas giraron en torno a (1) trayectoria del entrevistado en la TV comunitaria; (2) efectos de la normativa (Resolución 433 de 2013 y TLC entre Colombia y Estados Unidos) y (3) futuro de esta TV de proximidad, con base en la Resolución vigente, la 650 de 2018.

\section{Resultados}

4.1 Influencia del Tratado de Libre Comercio (TLC) entre Colombia y Estados Unidos y la Resolución 433 de 2013 de la Autoridad Nacional de Televisión (ANTV)

El funcionamiento y sostenibilidad de la Asociación de Usuarios Comunitarios de la Antena Parabólica de San Jorge de Ocaña, Asucap San Jorge (Colombia) se afecta, sin duda, por el TLC y la Resolución 433 de 2013, puesto que la obliga, así como a todos los sistemas comunitarios, a reducir de 15.000 a sólo 6.000 asociados que pueden pagar por ver. En Colombia, la disminución del número de asociados entre el 2012 y 2017 ha sido del 73,8\%, pues pasaron de tener 439.122 en el 2012 (cuando estaba vigente el Acuerdo 009 de 2006) a sólo 114.849 (con la Resolución 433 de 2013) (ANTV, 2018).

En el caso de Asucap, se llegó a tener un tope de 17.000 asociados [4], alrededor de 9.000 en el 2018 (Jácome, 2019), lo que representa una caída del $47 \%$ y dejar de percibir 152 millones de pesos mensuales (48.764 dólares estadounidenses )[5].

De otra parte la sentencia del Consejo de Estado del 2 de marzo de 2001 (Legis, 2001), causa polémica ya que beneficia al capital privado transnacional de la televisión por suscripción y por internet, señala que se deben evitar "prácticas monopolísticas en el uso del espectro electromagnético" contrario a lo que se indica en los artículos 75 y 76 de la Constitución Nacional, con los que se preserva el objetivo de la televisión comunitaria de "alcanzar fines cívicos, recreativos, culturales o institucionales".

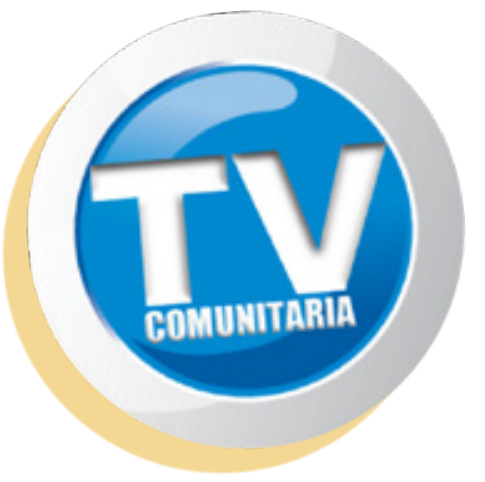




\subsection{Comparación entre el Acuerdo 009 de 2006 y la Resolución 433 de 2013}

La disminución a 6.000 asociados y, por ende, la restricción al ámbito geográfico y disminución de los ingresos económicos de Asucap San Jorge, no es la única decisión legal que influye en su funcionamiento y sostenimiento. En la siguiente tabla se aprecia cómo el cambio de la normativa de la Autoridad Nacional de Televisión (ANTV) (del Acuerdo 009 de 2006 a la Resolución 433 de 2013), afecta a esta televisión de proximidad.

\section{Tabla 2.}

\section{Comparación entre el Acuerdo 009 de 2006 (derogado) y la Resolución 433 de 2013 (derogado)}

\begin{tabular}{|c|c|c|}
\hline Acuerdo 009 de 2006 & Resolución 433 de 2013 & Observaciones \\
\hline $\begin{array}{l}\text { La cobertura del operador de TV } \\
\text { comunitaria no podrá ser superior a } \\
15.000 \text { asociados (Articulo 11). }\end{array}$ & $\begin{array}{l}\text { Para dar cumplimiento al TLC entre } \\
\text { Colombia y Estados Unidos, la TV } \\
\text { comunitaria sólo se puede prestar a } \\
\text { maximo } 6.000 \text { asociados (Artículo 10). }\end{array}$ & $\begin{array}{l}\text { La reducción del número de asociados, } \\
\text { obviamente le disminuye los ingresos a la } \\
\text { comunidad organizada. }\end{array}$ \\
\hline $\begin{array}{l}\text { Las TV comunitarias podrón distribuir } \\
\text { hasta siete (7) señales codificadas } \\
\text { (Articulo 7). }\end{array}$ & $\begin{array}{l}\text { Las TV comunitarias podrán recibiry } \\
\text { distribuir hasta siete (7) seศ̂ales } \\
\text { codificadas (Articulo 15). }\end{array}$ & $\begin{array}{l}\text { Es notoria la desventaja de las } \\
\text { comunidades organizadas frente a las TV } \\
\text { por suscripción pues pueden ofertar estos } \\
\text { canales de manera ilimitada. }\end{array}$ \\
\hline $\begin{array}{l}\text { Las TV comunitarias deben pagar el } \\
7 \% \text { de los aportes de los asociados } \\
\text { (Artículo 14). }\end{array}$ & $\begin{array}{l}\text { Serd el resultado de multiplicar el } \\
\text { número total de asociados al mes por } \\
\text { el Valor de Compensación por } \\
\text { Asociado al Mes (Articulo 12). }\end{array}$ & $\begin{array}{l}\text { En terminos sencillos, las comunidades } \\
\text { organizadas deberán pagar mús a la ANTV } \\
\text { con base en un porcentaje por cada } \\
\text { asociado. }\end{array}$ \\
\hline $\begin{array}{l}\text { De una hora a tres horas semanales } \\
\text { de producción propia, dependiendo } \\
\text { del número de asociados y señales } \\
\text { codificadas (Arviculo 17). }\end{array}$ & $\begin{array}{l}\text { Desde cinco a nueve horas } \\
\text { dependiendo del tiempo de } \\
\text { funcionamiento y no del número de } \\
\text { asociados (Articulo 16). }\end{array}$ & $\begin{array}{l}\text { Se estandariza la producción propia. El } \\
\text { error estriba en que no se le puede pedir } \\
\text { cinco horas de producción semanal a un } \\
\text { municipio de } 3.000 \text { habitantes que a otro } \\
\text { de } 150.000 \text {. }\end{array}$ \\
\hline
\end{tabular}

Fuente: Elaboración propia

\subsection{Decrecimiento de las televisiones comunitarias}

Uno de los efectos más notorios del TLC entre Colombia y Estados Unidos y la Resolución 433 de 2013 es el decrecimiento de las televisiones comunitarias en el país. En el siguiente gráfico se observa que a partir del año 2014, los sistemas comunitarios de Colombia y del departamento de Norte de Santander, donde opera Asucap San Jorge, han venido desapareciendo de los registros de la Autoridad Nacional de Televisión (ANTV) o han migrado a televisión por suscripción o por internet.

\section{Grafico 1.}

\section{Comportamiento cuantitativo de las TV comunitarias en Colombia y el departamento de Norte de Santander}

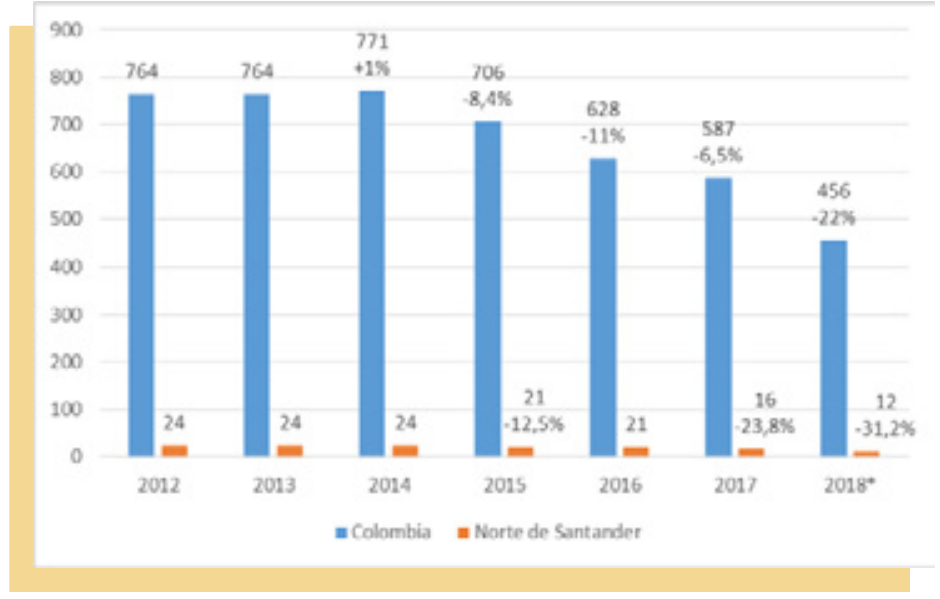


En Colombia entre los años 2014 y 2018, el $41 \%$ de estas televisiones de proximidad desaparecieron. En el departamento Norte de Santander el acumulado fue del $50 \%$. Es preciso recalcar que el modelo de televisión comunitaria y, por ende, el de Asucap San Jorge Ocaña, se financia con publicidad y por el pago mensual de sus asociados, mientras que modelos como los de Venezuela, Bélgica, Estados Unidos, Canadá, Holanda y Brasil son subsidiados por los Estados o por la televisión por suscripción (Zabaleta et al.,1998; Chaparro 2002; Krohling 2003; Conatel, 2018).

\subsection{Desaparición de las TV comunitarias y el déficit de información local}

El descenso cuantitativo de las TV comunitarias $y$, en consecuencia, del número de sus asociados, se puede unir al fenómeno del déficit de información local. Esto fue diagnosticado por el centro de investigaciones de la Fundación para la Libertad de Prensa (Flip) entre los años 2015 y 2017, con base en la revisión de 690 municipios colombianos. La conclusión fue que en el $56,8 \%$ de estas localidades se presenta déficit de información local. En el departamento de Norte de Santander -donde opera Asucap San Jorge- es del 60\% (Flip, 2017).

Si la desaparición de las TV comunitarias sigue la misma tendencia, como también puede ocurrir con las radios y prensa escrita análoga y virtual, lo más probable es que el déficit de información local sea aún mayor en el corto y mediano plazo.

\subsection{Perspectivas con la Resolución $\mathbf{6 5 0}$ de 2018}

La nueva Resolución que deroga la Resolución 433 de 2013 presenta cambios trascendentales para el funcionamiento y sostenimiento de la televisión comunitaria en Colombia. Uno de ellos como ya hemos referido en párrafos anteriores es el hecho de que sólo se podrá tener como máximo seis mil (6.000) asociados, en concordancia con lo dispuesto por el Tratado de Libre Comercio entre Colombia y Estados Unidos (Artículo 14). Aunque la Resolución vigente, la 650 de 2018 brinda la posibilidad de modificaciones o ampliaciones a esa cobertura, siempre y cuando el licenciatario de la TV comunitaria informe a la ANTV y remita copia del plano esquemático, en el que no puede superar los límites geográficos del respectivo municipio. También se puede superar la cifra de más de 6.000 asociados siempre que la programación cumpla el número de horas exigidas.

La reducción delacobertura pod ría provocar que empresas de internet no acreditadas ante la ANTV ofrezcan televisión por redes sociales directamente a los celulares, en tal caso los usuarios deberán asumir los costos por utilización de su plan de datos. Esa normatividad beneficia a los operadores de la TV por suscripción ya que es posible que el asociado comunitario que pueda pagar TV por dicha modalidad se pase dejando de lado la TV comunitaria. Un ejemplo claro de ello es Asucap San Jorge Ocaña que se debilitó económicamente con la disminución de sus asociados; al generar menos ha debido reducir la planta de personal y las horas semanas de producción propia; lo que ha afectado la calidad de la programación.

Otro cambio es el del valor de la compensación. En la Resolución 433 de 2013 se establecía que el sistema comunitario debía pagar a la ANTV un porcentaje por cada asociado, la Resolución 650 señala que este valor se debe calcular con base en los ingresos brutos mensuales provenientes de la explotación del servicio de TV y de acuerdo con el tamaño del municipio (Artículo 16), así: poblaciones entre 1 y 20.000 habitantes, deberán pagar el 0,20\% de los ingresos brutos mensuales; municipios que tengan entre 20.001 y 100.000 habitantes, el $0,40 \%$ y las localidades con más de 100.001 habitantes, el 4\%. Cuando los ingresos brutos mensuales sean superiores a 127 salarios mínimos legales vigentes en Colombia [5], el valor de la compensación será del 5,9\%, independientemente del número de habitantes.

En relación con los pagos por compensación el costo es muy alto, puesto que adicionalmente de lo que se le tiene 
que pagar a la ANTV por la prestación del servicio, también hay que pagar el valor de los canales codificados que se emiten. Por ejemplo, hay canales como Winsports por donde se transmite el fútbol profesional colombiano que son muy caros y no se pueden suministrar, pues valen 5.000 pesos colombianos por persona.

Un cambio igualmente trascendente es el que concierne a las horas de producción propia. La Resolución vigente estipula que se deban producir entre tres y seis horas semanales, dependiendo del número de asociados y no del tiempo que lleve funcionando el canal comunitario. El problema que indicamos en la tabla 2, es que la Resolución anterior, la 433 de 2013, estandarizaba la producción propia de una TV comunitaria (entre cinco y nueve horas semanales) que operaba tanto en un municipio de 4.000 habitantes, por ejemplo, como en otro de 150.000 con más vida económica, política y cultural.

En relación a las dificultades para producir programación propia semanal: Asucap San Jorge Ocaña cuenta con una de las mejores programaciones del país, prueba de ello son los premios ganados en los órdenes nacional y regional. Aun así, faltan recursos económicos para contratar más personal, con mejores salarios y más calificados que mantengan una programación de calidad. También faltan directrices claras de producción audiovisual comunitaria.

Cuántos canales codificados deberían ofertar los sistemas comunitarios: Este es un punto determinante porque los asociados piden más canales codificados de calidad y el pago de los derechos de autor por cada uno de ellos es alto, situación que se transfiere al costo del servicio del usuario. Sin embargo, existe la barrera de los siete canales codificados. La idea es que se puedan ofertar en forma ilimitada y por medio de codificación (set top box) establecer planes para los segmentos de la población con distintos ingresos y estratos socioeconómicos.

\section{Conclusiones}

En los últimos cinco años Asucap San Jorge de Ocaña y las televisiones comunitarias del país han funcionado en un contexto normativo adverso. La reducción de su cobertura, esto es, la reducción del número de asociados de 15.000 a 6.000, medida contemplada en la sentencia del Consejo de Estado y transferida al espíritu del TLC entre Colombia y Estados Unidos y la Resolución 433 de 2013, vigente hasta el año 2018, sin duda limitó el desarrollo y sostenibilidad de los sistemas comunitarios. Al detenerse en los términos de la sentencia, se evidencian situaciones contradictorias que vale la pena señalar.

Primero, la sentencia indica que su texto es coherente con los Artículos 75 y 76 de la Constitución Nacional porque se evitan prácticas monopolísticas de los operadores comunitarios. Sin embargo, ¿se podría hablar de monopolios del servicio de TV por parte de comunidades organizadas sin ánimo de lucro con escaso capital económico y financiero que van a municipios donde no hay información local? Aquí es justo recordar el informe de la Fundación para la Libertad de Prensa (Flip, 2017), en el que se concluye que, de los 690 municipios rastreados del país, el $56,8 \%$ presenta déficit de información local y de los 40 municipios de Norte de Santander, departamento donde opera Asucap San Jorge, el porcentaje es del $60 \%$.

Es probable, por tanto, que haya una conexión entre desaparición de sistemas comunitarios con la no existencia de medios o de información local. Adicionalmente, la sentencia dice que la disminución del ámbito geográfico tiene por objeto evitar que se desnaturalicen los fines cívicos, recreativos y culturales o institucionales de la TV comunitario al extenderlos a otros sectores. Se pensaría, entonces, que dichos fines sólo son exclusivos de comunidades organizadas de bajos 0 medios ingresos económicos y no pueden serlo de otros sectores con más ingresos y otras expectativas. Tal interpretación es a todas luces errónea. 
El panorama también es adverso, puesto que fuerza a las TV comunitarias a migrar a formatos como los de la TV por suscripción o por internet, los cuales sí persiguen el lucro y no es su interés producir una programación educativa y cultural. Todo ello sugiere que los más beneficiados son las TV por suscripción y por internet, pues los asociados buscarán donde ver televisión con canales codificados, aunque carezcan de un canal comunitario de calidad.

Consecuencias del TLC entre Colombia y Estados y la Resolución 433 de 2013 en el funcionamiento de las TV comunitarias, particularmente de Asucap San Jorge Ocaña. Las consecuencias de la Resolución 433 de 2013 fueron la desaparición del número de televisiones comunitarias en el país y la reducción significativa de los ingresos de estos sistemas por la salida de un poco más de 324.000 asociados entre el 2012 y 2017 (ANTV, 2018), los cuales habrían pasado a la TV por suscripción o se habrían quedado sin televisión cerrada. En Colombia, por ejemplo, un $41 \%$ de estos medios de comunicación dejaron de existir y en el departamento Norte de Santander, donde opera Asucap San Jorge, el porcentaje fue más alto, $50 \%$, fenómeno que pudo incidir en el déficit de información local diagnosticado por la Fundación para la Libertad de Prensa (2017).

Asucap también se perjudicó sensiblemente con la pérdida de 8.000 asociados en el mismo periodo, lo cual le representó, por un lado, dejar de percibir dineros para la reinversión en equipos $y$ el sostenimiento $y$, por otro, pagar un valor más alto por compensación por los usuarios que se quedaron en el sistema comunitario. En concreto, como lo señalan los entrevistados, se tuvo que reducir la planta de personal, la producción propia de contenidos semanales y la calidad de la parrilla de programación.

Estos hechos repercuten negativamente en la esencia del enfoque comunitario, puesto que las personas no tienen programas de excelencia en los que puedan expresar la vida política, económica y social de su municipio, divulgar la cultura, promover campañas de beneficio colectivo y ser garante del patrimonio histórico.

Perspectivas con la Resolución 650 de 2018. Son cuatro aspectos fundamentales de la Resolución vigente. El primero se refiere a mantener la restricción geográfica derivada de autorizar que cada sistema comunitario sólo tenga, como máximo 6.000 asociados. Sin embargo, el parágrafo del Artículo 14 prevé que, si ese número se sobrepasa, se debe informar y remitir plano esquemático a la ANTV. En otras palabras, es probable que la Autoridad Nacional de Televisión apruebe una cantidad mayor, siempre que no supere el ámbito geográfico del municipio y, como lo aseguró uno de los entrevistados, se cumpla con el número de horas de producción propia semanal.

A pesar de esa supuesta concesión, lo claro es que superar los 6.000 asociados se convierte en algo excepcional, sujeto al estudio de la ANTV, y no queda normado como ocurría con el derogado Acuerdo 009 de 2006 que aceptaba 15.000. La consecuencia salta a la vista: la TV comunitaria pierde competitividad frente a la TV por suscripción y se priva a usuarios de escasos recursos económicos a tener una televisión cerrada de calidad con su respectivo canal comunitario.

El segundo concierne al pago de compensación que, a priori, beneficia a las TV comunitarias al no tener que pagar un porcentaje por cada asociado, dependiendo de las condiciones económicas del municipio donde reside (necesidades básicas insatisfechas), sino por el número de asociados.

El tercer aspecto fundamental de la Resolución 650 de 2018 se centra en el número de canales codificados que se pueden ofertar. Aquí todas las normativas recientes estipulan que sólo siete señales de este tipo se pueden transmitir, con lo cual se limita la competitividad de la TV comunitaria. La opción que se debe mirar a futuro es que la oferta sea ilimitada $y$, 
como lo afirmó uno de los informantes, se pongan a consideración paquetes de canales codificados, mediante la utilización la tecnología set top box, para personas con distintos ingresos económicos.

El cuarto guarda relación con la cantidad de horas de producción propia semanal. La Resolución 650 de 2018 corrige el error de la Resolución 433, según el cual el número de horas dependía del tiempo de funcionamiento, lo cual implicaba que un sistema comunitario, con cuatro mil habitantes, escasos asociados y poca actividad política, económica y cultural debía llegar a nueve horas de programación semanal. En cambio, la Resolución 650 tiene en cuenta las diferencias entre localidades $y$, por tanto, configura que la producción propia sea por número de asociados y hasta seis horas semanales. Pese a que la medida es congruente con las condiciones de la organización comunitaria, no resuelve el problema de fondo, esto es, la falta de capacitación audiovisual para la elaboración de programas comunitarios de calidad, tarea pendiente de la ANTV.

\section{Referencias}

Ángulo, L. (2015). Televisión comunitaria: producción, programación y audiencia. Luciérnaga Comunicación. 5 (10), 64-77. Recuperado de https://revistas.elpoli. edu.co/index.php/luc/article/view/311

Autoridad Nacional de Televisión. (8 de junio de 2018). Resolución 650 de 2018. Reglamentación del servicio de televisión comunitaria. Obtenido de antv.gov.co: https://goo.gl/X7QqhX

Autoridad Nacional de Televisión. (21 de mayo de 2018). Directorio operadores de la TV comunitaria. Obtenido de ANTV: https://goo.gl/2gxKrp

Autoridad Nacional de Televisión. (20 de mayo de 2018). Asociados de TV comunitaria cerrada sin ánimo de lucro. Obtenido de ANTV: https://goo.gl/ pV9HD4

Autoridad Nacional de Televisión. (1 de diciembre de 2013). Resolución 433 de 2013. Obtenido de ANTV: https://goo. $\mathrm{gl} / \mathrm{xaFR59}$

Autoridad Nacional de Televisión. (26 de octubre de 2006). Acuerdo 009 de 2006. Obtenido de ANTV: https://goo. gl/Nt3dRW

Chacón, J. (2017). Características de Los Medios Comunitarios y Alternativos de Bogotá - Colombia. Luciérnaga, 8(15), 63-83. doi:https://doi.org/10.33571/ revistaluciernaga.v8n $15 \mathrm{a} 5$

Chaparro, M., (2002). Sorprendiendo al futuro. Comunicación para el desarrollo e información audiovisual. Primera ed. Barcelona: Los libros de la frontera (Comunicación).
Comisión Nacional de Telecomunicaciones de Venezuela, (2002). Reglamento de radiodifusión sonora y televisión abierta comunitarias de servicio público, sin fines de lucro. obtenido de: https://goo.gl/pvRKHU

Dagron, A. G. (2011). Comunicación para el cambio social: clave del desarrollo participativo. Signo y Pensamiento(58), 26-39.

Dagron, G. A., (2002). TV comunitaria: ni púlpito, ni pulpo: pálpito. Obtenido de: https://goo.gl/awA3AW [Último acceso: 31 de mayo 2018].

Fundación para la Libertad de Prensa. (1 de diciembre de 2017). Cartografía de la información. Obtenido de El periodismo local se extingue en Colombia: https:// goo.gl/wwPZHc

Garcia, A. P. (2012). Televisión en Colombia: Surgimiento de los canales regionales. Revista Virtual Luciérnaga. Grupo de investigación en Comnicación, Facultad de Comunicación Audiovisual, Politécnico Jaime Isaza Cadavid, 4(7), 23-35. Obtenido de https://revistas. elpoli.edu.co/index.php/luc/article/ view/283

Hernández, R., \& Fernández, C. y Baptista, P. (2010). Metodología de la investigación. Quinta edición. Lima, Perú: McGraw Hill.

Krohling, C., (2003). La TV comunitara en Brasil: aspectos históricos. Obtenido de: https://goo.gl/EVZfbX [Último acceso: 21 de mayo 2018]. 
Legis (2 de marzo de 2001). Sentencia del Consejo de Estado 1999-05907 del 02 de marzo de 2001. Obtenido de Legis: https://goo.gl/JgQTf8

McQuail, D. (2000). Introducción a la teoría de la comunicación de masas. Barcelona: Paidós.

Ministerio de Comercio.(12 de diciembre de 2007). Mincomercio publica texto y anexos del TLC con Estados Unidos. Obtenido de Mincomercio: https://goo. $\mathrm{gl} / \mathrm{fqC} \mathrm{kv} 7$

Servaes, J., Polk, E., Shi, S., \& Reilly, D. \& Yakupitijage, T. (21 de febrero de 2012). Towards a framework of sustainability indicators for 'communication for development and social change' projects. Obtenido de International Communication Gazette: https://goo.gl/ FbV8TD

Soler, P. (2011). La investigación cualitativa. Un enfoque integrador. En L. (. Vilches, La investigación en comunicación: métodos y técnicas en la era digital (págs. 189-236). Barcelona: Gedisa.
Toro, I. D. y Parra, R. D. (2010). Fundamentos epistemológicos de la investigación y la metodología de la investigación cuantitativa/cualitativa. Medellín: Fondo Editorial Universidad Eafit.

Valle, M. (2015). Medios alternativos de Medellín [1]: Recuento Histórico (1987-2006). Luciérnaga Comunicación. 4(7), 36-46. DOI: https://10.33571/ revistaluciernaga.v4n7a4 . Disponible en: https://revistas.elpoli.edu.co/index. php/luc/article/view/284

Valle, M. (2015). Medios Alternativos de Medellín [2]: Diagnóstico. Revista Virtual Luciérnaga. Grupo de Investigación en Comunicación, Facultad de Comunicación Audiovisual, Politécnico Jaime Isaza Cadavid, 4(7), 51-57. https://10.33571/revistaluciernaga. v4n8a3 . Recuperado de https://revistas. elpoli.edu.co/index.php/luc/article/ view/1510/1134

Weerakkody, N. (2009). Research Methods For Media And Communication. Sidney (Australia): Oxford.

\section{Notas}

[1] Entidad que regula la televisión en el país.

[2] La Comisión Nacional de Televisión fue reemplazada por la Autoridad Nacional de Televisión. [3] La expresión "Departamento" es equivalente a "Estado", Provincia o "Comunidad Autónoma" en otros países.

[4] Incluso 2.000 asociados más de lo estipulado en el derogado Acuerdo 009 de 2006 (15.000). [5]El dólar se cotiza a 3.185 pesos colombianos a 17 de julio de 2019.

[6]La cifra sale de multiplicar los 8.000 asociados que salen de la TV comunitaria por los 19.000 pesos colombianos, correspondientes al valor que deben por el servicio de Asucap San Jorge. [7]Equivalente a \$105.170.732 del año 2019 (33.676 dólares estadounidenses).

\section{Para citar:}

Angulo Rincón, Lizandro y Páez Quintana, Heverd (2019). MEDIOS ALTERNATIVOS. Panorama de la normatividad de la televisión comunitaria. Revista Luciérnaga Comunicación. Vol. 11, Núm. 22. Pp 146 - 158.

Doi: https://doi.org/10.33571/revistaluciernaga.v11 n22a7

OJS. https://revistas.elpoli.edu.co/index.php/luc/issue/archive 